\title{
Acoustofluidics 9: Modelling and applications of planar resonant devices for acoustic particle manipulation
}

\author{
Peter Glynne-Jones, ${ }^{a}$ Rosemary J. Boltryk ${ }^{a}$ and Martyn Hill*a \\ Received (in $X X X, X X X)$ Xth $X X X X X X X X X 20 X X$, Accepted Xth $X X X X X X X X X 20 X X$ \\ ${ }_{5}$ DOI: 10.1039/b000000x
}

This article introduces the design, construction and applications of planar resonant devices for particle and cell manipulation. These systems rely on the pistonic action of a piezoelectric layer to generate a one dimensional axial variation in acoustic pressure through a system of acoustically tuned layers. The resulting acoustic standing wave is dominated by planar variations in pressure causing particles to migrate

10 to planar pressure nodes (or antinodes depending on particle and fluid properties). The consequences of lateral variations in the fields are discussed, and rules for designing resonators with high energy density within the appropriate layer for a given drive voltage presented.

\section{Introduction}

There is a need to manipulate micron-scale particles and cells in many areas of physics, analytical chemistry and the biosciences. ${ }_{15}$ Techniques such as filtration, centrifugation and sedimentation are well-established in macro-scale applications, but in microfluidic systems the use of other approaches including optical, magnetic, dielectrophoretic and acoustic forces are of interest. Acoustic radiation forces, typically at ultrasonic frequencies in the hundreds of $\mathrm{kHz}$ to tens of $\mathrm{MHz}$ region, have wavelengths that are well matched to microfluidic channel scales, yet are capable of generating potential wells with significantly larger length scales. The technology is also relatively straightforward to integrate within microfluidic systems.

\section{Acoustic radiation forces}

Acoustic radiation forces can be generated on particles by both travelling and standing acoustic fields. Those in standing wave fields (of primary interest in the applications considered here) are generated by the nonlinear interaction between the acoustic field scattered by the particle and the standing wave field itself. The time averaged radiation force $F(\boldsymbol{r})$ on a small (in comparison with a wavelength) spherical particle of volume $V$ located at $\boldsymbol{r}$ within a stationary acoustic field was shown by Gor'kov ${ }^{1}$ to relate to the gradients of the time 25 averaged kinetic and potential energy densities ( $E_{k i n}$ and $E_{p o t}$ respectively) within the field:

$$
F(\boldsymbol{r})=V \nabla\left(\frac{3\left(\rho_{p}-\rho_{f}\right)}{\left(2 \rho_{p}+\rho_{f}\right)} E_{k i n}(\boldsymbol{r})-\left(1-\frac{\beta_{p}}{\beta_{f}}\right) E_{p o t}(\boldsymbol{r})\right)
$$

The kinetic energy density gradient (a function of the acoustic velocity field within the standing wave) is weighted by a function of the densities $\left(\rho_{p}\right.$ and $\rho_{f}$ ) of the particle and the surrounding fluid respectively. The potential energy density gradient (a function of the acoustic pressure within the standing wave) is weighted by a function of the compressibilities $\left(\beta_{p}\right.$ and $\beta_{f}$ ) of the particle and the 30 surrounding fluid. Most particles and cells of interest are denser and less compressible than typical suspending fluid, so there is a force on them that tends to move them to the acoustic pressure node, and the acoustic velocity antinode. In a planar resonator these are colocated.

\section{Generating the required acoustic field}

Many approaches to generating the required acoustic wave field have been reported in the literature. These include the use of near-field 35 effects ${ }^{2}$ or focussed ultrasound ${ }^{3,4}$ to trap particles and cells, the excitation of lateral standing waves in which the predominant energy gradients run parallel to the face of the excitation transducer ${ }^{5,6}$, and the generation of cylindrical resonances to focus ${ }^{7}$ or arrange 
particles $^{8}$. Particles can be held and moved within standing waves excited by plate waves coupled into the containing fluid ${ }^{9,10}$ and there is currently significant interest in the use of surface acoustic waves ${ }^{11-13}$ or interface waves ${ }^{14}$ to provide acoustic energy within microfluidic channels.

The most straightforward approach to establish a standing wave, however, is to use a planar, layered resonant device which, to a first 5 approximation, establishes a one dimensional resonance pattern along the axis of the outgoing and returning acoustic field ${ }^{15-17}$. A number of investigators have employed a planar design incorporating pairs of opposing transducers to modulate the standing wave field 18,19 , but this paper focuses on planar systems with a single transducer.

\section{Modelling of planar resonators}

${ }_{10}$ For the purposes of obtaining insight into device operation, and to provide high efficiency numerical formulations to enable parameter space exploration for device design, a planar resonator can be approximated as a 1D device. Fig. 1 shows a typical configuration composed of a sequence of different layers. The piezoelectric transducer couples acoustic energy into a carrier layer. The carrier layer is also sometimes described as a matching layer. In the context of travelling waves the function of a matching layer is to couple energy more efficiently into subsequent layers, however in the case of a resonant system the role of this layer is less straightforward, and such 15 tuning can also be accomplished by suitable choice of the other layers (as demonstrated below). In this case the function of the carrier layer may be more structural, or to isolate the transducer from the fluid layer. The fluid layer is the region in which the particle manipulation is to occur, and the reflector layer serves to reflect energy back into the device, and hence maintain strongly resonant operation. At both ends of the device the layers are usually backed by air. The glue layer used to bond the transducer to carrier layers can also have a significant effect on device operation, especially in the quarter-wave devices described below.

20 The sequence of layers after the transducer can be modelled using an approximation of 1D linear acoustic propagation through the layers, and models have been described in a variety of forms ${ }^{17,20,21}$, Aside from varying degrees of accuracy in the transducer representation (see below), the approaches such as the "transfer impedance", "transfer matrix", and "equivalent circuit models" are all mathematically similar. These models typically consider the system of layers as analogous to a set of electrical transmission lines. For an excellent discussion of one dimensional modelling for the design of resonators of multiple wavelengths in size, readers are referred to 25 the series of papers by Gröschl ${ }^{15,22}$. A worked example can be found in part 2 of this series ${ }^{23}$.

The representation of the transducer driving the layers can be a simple forcing function at the carrier layer boundary. However this ignores the coupling between the finite impedance transducer and the remainder of the resonator. It is more physically accurate to use a representation such as the Mason model (or the commonly used KLM model). These use an equivalent circuit to model the electroacoustic response of a thickness mode resonance in a piezoelectric plate (other types of resonance mode can also be represented with 30 alternative model parameters). The Mason model configuration is presented in Fig. 2; the values of the parameters can be found elsewhere ${ }^{24}$. The Mason model is derived from the constitutive piezoelectric relations described in part 4 of this tutorial series ${ }^{25}$. The $\mathrm{KLM}_{\text {model }}{ }^{26}$ was developed as an easier-to-use alternative to the Mason model; its layout is similar, but incorporates a transmission line in place of $Z_{T}$, and reduces the number of components. It has been shown to produce similar results to the Mason model under typical boundary conditions if care is taken to apply complex loss values consistently ${ }^{24}$. However comparing the response of the two 35 models to an electrical impulse, a time-delay is seen in the output of the KLM model as the impulse propagates through the transmission line. Having deduced the pressure distribution within the fluid layer, equation (1) above can be used to predict the radiation force on particles in the resonant field.

An alternative method for predicting device performance is to use a finite element analysis (FEA) package such as ANSYS or COMSOL to represent the various layers, and solve the resulting linear equations ${ }^{27-29}$. These packages are able to model the 40 piezoelectric interactions using the full constitutive relations ${ }^{25}$, and couple the resulting acoustic field into a detailed structural model of the device components. Once again, by including equation (1) above in a post-solution step, it is possible to create an FEA model that predicts radiation forces from the geometry, electrical drive and material properties.

By using suitable symmetry conditions a 1D model can be created; Fig. 3 compares the transfer impedance model with a KLM transducer representation to an ANSYS FEA model for a typical quarter-wave configuration (presented elsewhere ${ }^{30}$ ), and shows good 45 agreement. Small inconsistencies are to be expected due the differences in the ways the models implement the damping and transducer representation.

The 1D approach is an approximation that ignores the effect of lateral resonances within the various layers, plate waves, surface waves, and edge effects. In practice, significant departures can be seen from the ideal ${ }^{31}$ and this can be observed in Fig. 4 , which uses an ANSYS FEA model to predict the field in a silicon microfabricated filtration device ${ }^{27}$ (this is an early FEA model that uses a pressure 50 boundary condition rather than a finite element transducer representation). In such devices the following non-ideal phenomena are typically seen: (a) width-wise variation in node position - the height of the node in the fluid channel often shows a corrugated pattern (as shown in Fig. 4), (b) width-wise variation in focusing force (particularly a reduction in force near edges), (c) width-wise forces that caused particles to form into bands, and (d) trapping forces capable of holding particles and agglomerates against a flow. In longer devices with particle flow over an acoustic field region, these effects may be less pronounced due to an averaging of the differing 55 acoustic environments. However the averaging effect is not perfect, particularly for lower flow rates. Frequency sweeping can also reduce these effects by effectively averaging in time the force-fields of similar acoustic modes ${ }^{32}$. 


\section{Resonator configurations}

Any combination of layer thicknesses will show acoustic resonances at certain frequencies; some configurations will be much more strongly resonant than others, and it is these that are discussed here, as the increased energy density resulting from the resonance makes the devices more useful in practice. It should also be noted that devices can be run at frequencies other than their resonant frequency 5 this is rarely useful in lightly damped devices as the energy density decreases rapidly away from resonance.

Layered resonators can be classified into a number of categories based upon the number of wavelengths and position of nodes within the various layers. The distinctions described here are popularly used in the literature, and are useful as each class displays distinct characteristics. In devices made with low loss materials the distinctions are clear; however in devices made from more lossy polymeric materials the distinctions are less clear, and since layer thicknesses can vary continuously there are designs that do not fit easily into the 10 classification. Although these devices are usually less energy dense, they have the advantage that the node position is more flexible (i.e. shifts in drive frequency can move the nodal position) and less determined by the geometry of the fluid layer ${ }^{33}$. Hawkes ${ }^{34}$ explores a range of configurations, some of which are described below, based upon layer thicknesses that are multiples of $\lambda / 4$. Fig. 5 shows typical pressure distributions in the various classes of device.

15

\section{Half wave devices}

Half wave devices can be defined as resonators in which the fluid channel is close to a half wavelength in thickness. For systems with a reflector that has higher acoustic impedance than the fluid, this leads to a resonance across the thickness of the channel, with a pressure minimum close to the channel centre. This mode is thus useful for focusing particles into a plane away from the chamber walls.

20 Generating half wavelength modes at high ultrasonic frequencies, implies sub millimetre chamber depths and such chambers were investigated by Hawkes et al. ${ }^{35}$ for particle filtration ${ }^{16}$ and cell washing ${ }^{36}$.

The transfer impedance model mentioned above ${ }^{17}$ can be used to explore the parameter space for the optimum selection of layer thicknesses for a planar half-wave design. The following optimisation is based on a design with a PZT4D transducer (speed of sound, $c=4530 \mathrm{~m} \mathrm{~s}^{-1}$, density, $\rho=7700 \mathrm{~kg} \mathrm{~m}^{-3}$, piezo-constant $h_{33}=2.37 \mathrm{e} 9 \mathrm{~V} \mathrm{~m}^{-1}$, relative permittivity $\varepsilon_{r}=700$ ), and carrier and reflector layers of 25 glass $\left(\rho=2240 \mathrm{~kg} \mathrm{~m}^{-3}, c=6000 \mathrm{~m} \mathrm{~s}^{-1}\right)$, and water as the fluid $\left(\rho=1000 \mathrm{~kg} \mathrm{~m}^{-3}, c=1480 \mathrm{~m} \mathrm{~s}^{-1}\right)$. The damping was approximated by a Qfactor (the ratio of the stored to dissipated energy) of 100 in all layers (this also accounts for other losses in the structure). The energy densities quoted below are for a drive voltage of $10 \mathrm{Vpp}$. While the results are specific to these materials, the optimisation shows some useful design principles that could be extended to other material systems.

The PZT thickness was fixed at $1 \mathrm{~mm}$ (see discussion of scaling, below). For each combination of parameters, the frequency with the 30 maximum radiation force was found, as shown in Fig. 6. In order to visualise the optimisation of the three variables Fig. $\mathbf{6}$ is plotted first for the case when there is no carrier layer, then for a $\lambda / 4$ reflector layer. It can be seen that the optimum combination is for a fluid layer of $360 \mu \mathrm{m}$, reflector layer $800 \mu \mathrm{m}$ thick, and zero carrier layer. At the resonant frequency of this combination, $1.90 \mathrm{MHz}$, these can be expressed as fractions of the acoustic wavelength in their corresponding layers: transducer $0.84 * \lambda_{\text {xdr }} / 2$; fluid layer $0.93 * \lambda_{\mathrm{f}} / 2$; reflector thickness $1.0 * \lambda_{\mathrm{r}} / 4$.

35 In a previous paper ${ }^{37}$ the variations of carrier thickness were explored for a fixed fluid layer of $\lambda / 2$ thickness, leading to the conclusion that a non-zero carrier layer thickness was optimum. This fixed fluid layer case is supported here, but we see that a stronger radiation force is found for a thinner fluid layer and zero carrier layer thickness. The optimum carrier layer thickness is also different to that suggested by Gröschl ${ }^{22}$, which was for an analysis where the operating frequency was constrained to be identical to the resonance frequency of the free transducer.

40 The optimum found here (the highest force for a given transducer drive voltage) is not very sensitive to variations: reflector thickness can vary by $\pm 60 \%$ to cause a drop of only $10 \%$ in radiation force; similarly, fluid layer thickness can vary by $\pm 10 \%$ for the same drop. When the reflector thickness is close to $\lambda / 2$ the radiation force becomes much weaker (we can also question whether the resulting field pattern can really be called a half-wave design), but recovers effective operation even quite close to this.

If we explore this optimum point in more detail, we find that for these layer thicknesses there are two possible resonances, shown by 45 the twin energy density peaks in Fig. 7a. The corresponding pressure distributions through the layers for each of these resonances are also plotted; the higher frequency of the pair has a pressure node in the PZT which (since the radiation force is proportional to the gradient of the potential energy density and hence gradient of pressure amplitude squared, see Equation 1) will lead to a force towards the carrier layer near the bottom of the fluid layer; in contrast the lower frequency resonance tends to push particles away from the carrier layer. It is found that the lower frequency case is always more energetic in the region plotted in Fig. 6a,b. In addition to being stronger, 50 this lower frequency resonance is also more likely to be practically useful as it tends to drive (dense) particles away from the transducer/matching layer side of the fluid channel.

The effect of reflector thickness on the radiation force near the fluid/reflector boundary is shown in Fig. 6c. Interestingly, we find that we can take advantage of the insensitivity to reflector thickness to choose whether there is a force into the reflector, or away from it (positive force is into reflector). In many applications we want to ensure there is a force away from the reflector and will choose a

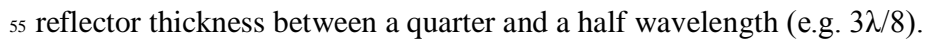

The above results are all for a PZT thickness of $1 \mathrm{~mm}$. However planar resonator designs can be scaled linearly - i.e. if the PZT thickness is doubled, the corresponding optimum thicknesses for the other layers are also doubled (and the operating frequency halved).

We can summarise the above findings in the following half-wave design rules: 
1) Make the carrier layer as thin as possible, or better, omit it entirely.

2) Optimum transducer thickness close to $\sim 0.84 * \lambda / 2$ (corresponding to a transducer running close to its own resonant frequency)

3) Optimum fluid thickness $\sim 0.93 * \lambda / 2$ (less if more damping, see below)

4) Optimum reflector thickness $\sim \lambda / 4$ (very insensitive)

5 5) For force away from reflector choose reflector thickness greater than $\lambda / 4$

6) For force away from carrier layer choose lower frequency resonance (of the two possibilities available for any given combination of layer thicknesses)

The above analysis is for an optimum radiation force for a given transducer drive voltage. In some applications where self-heating is the limiting factor, rather than drive voltage we may wish to optimise for the performance number as defined by Grösch ${ }^{15}$. This non10 dimensional metric is defined as the ratio of energy density in the fluid layer to unit electrical energy per cycle entering the system. We plot in Fig. 6d the performance number over a range of layer thicknesses, and see that the optimum region in this case is much less sensitive to fluid thickness.

The exact positions of the optimum dimensions will depend on material properties, however the trends above should be valid for materials with a high acoustic impedance. Q-factors in the range 50 to 500 were also explored, and similar trends found - the position of 15 the optimum radiation force or performance number was essentially unchanged. For a given frequency the force on a particle in the channel is proportional to the acoustic energy density (see Eq. 1). For these material parameters, the configuration with maximum energy density was found to always coincide with that of maximum radiation force. We should note that one may also wish to optimise for average force, or force at a particular boundary which may not coincide with this optimum set of layer thicknesses.

While the half-wave resonator has a fluid layer thickness of $\sim \lambda / 2$, similar configurations with thickness $\mathrm{n} \lambda / 2$ behave in a similar 20 manner, but with multiple trapping planes (see applications section, below).

Further considerations for the design of resonators are described in detail by Gröschl ${ }^{22}$, including the effects of lack of planarity, and acoustic absorption.

\section{Quarter-wave resonators}

Quarter-wave devices are characterised by a resonant reflector layer that effectively imposes a boundary release pressure condition at the 25 top of the fluid layer. They are designed to bring particles towards the reflector / fluid interface ${ }^{38}$.

In contrast to half-wave designs, quarter-wave devices are very sensitive to their reflector thickness; this is largely due to the necessity of tuning two resonances: the resonance across the transducer, carrier and fluid layers, and also the resonance in the reflector layer. Townsend et al ${ }^{39}$ show that for a typical design a change in the reflector thickness of $5 \%$ is sufficient to cause a drop in radiation force of $50 \%$. In addition, lateral effects (from non-ideal resonances that have a lateral variation), can cause the node location to vary between a 30 position in the reflector and a position within the fluid channel making it difficult to push particles uniformly onto the reflector surface. It is additionally found that an antinode can exist with the channel causing particles to move towards the carrier layer rather than the reflector depending on the initial position of the particle. The quarter-wave design is so sensitive to layer thicknesses that general design rules of the type describe above for a half-wave device are hard to form, and a 1D computer-based layer model is required for effective design.

35 It is also possible to have an inverted quarter-wave resonant mode in which the transducer and carrier layers are in resonance, coupled to a resonance across the fluid and reflector layers such that there is a node close to the carrier/fluid (boundary), pushing particles towards this interface.

\section{Thin-reflector resonators}

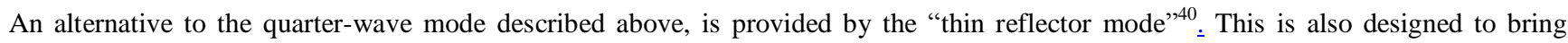
${ }_{40}$ particles towards the reflector/fluid interface. In this mode, the first order resonance of the whole layered structure is utilised. The reflector, fluid and carrier layers are all thin compared to the wavelength (e.g. 0.1 $\lambda$ ) such that particles in the fluid channel are attracted towards the pressure node at the reflector/air interface. In contrast to the quarter-wave mode, this mode is relatively insensitive to layer thickness variations, and when suitably designed there are positive forces towards the reflector/fluid interface from all positions within the fluid channel. Since relatively little of the acoustic energy is stored within the carrier or reflector layers, this mode is particularly 45 suitable for implementation in polymers to provide disposable devices.

Fig. 8 shows layer thickness optimisation for a thin reflector device using the same material properties as described above for a halfwave device and a $1 \mathrm{~mm}$ thick transducer. Again, it is found (graphs not shown) that having no carrier layer is the most efficient configuration for achieving both maximum average radiation force, and maximum performance number. The average radiation force (spatially averaged over the fluid layer) shows a broad maximum over a range of fluid layers having over $90 \%$ of its maximum value 50 over the range $t_{f}=0 \ldots 70 \mu \mathrm{m}$ (corresponding to zero to $0.08 * \lambda_{\mathrm{f}}$ ).

\section{Position control in resonators}

The resonant designs described above are limited in the flexibility with which the equilibrium particle position can be manipulated. The nodal position is designed by appropriate choice of layer thicknesses, and once created there is little scope for moving this position by, for example, changing frequencies as radiation force is lost away from the resonant frequency. The node position is essentially fixed by 55 the device geometry. A number of strategies have been demonstrated to overcome this limitation, and are described below. Some of

4 | Journal Name, [year], [vol], 00-00 This journal is (C) The Royal Society of Chemistry [year]


these are more applicable to manipulation in 2D and 3D devices and will be discussed in more detail in a forthcoming review paper in this series.

Kozuka et al. ${ }^{41}$ demonstrated an array of multi-wavelength focussed transducers acting in air, and showed that particles tended to become positioned above active elements. Demore et al ${ }^{42}$ integrated this into a microfluidic environment, and showed that this effect is 5 due to secondary, kinetic energy gradient driven forces.

If high radiation force acoustic fields are not required, then wider bandwidth polymer device layers ${ }^{33}$ increases the bandwidth of the device. This allows the resonator to be run over a range of frequencies and the axial nodal positions can then be adjusted simply by changing the driving frequency.

In multi-wavelength devices, sweeping the frequency in a saw tooth pattern enables particles to be moved continuously over a distance 10 of many wavelengths. ${ }^{9,32,41,43}$

An alternative approach has been demonstrated by Glynne-Jones et al. ${ }^{44}$. This allows continuous particle positioning in a device that supports both a half-wave and a quarter-wave mode. It was shown that by rapidly switching between the two modes at a sufficiently high frequency (e.g. $100 \mathrm{~Hz}$ ), provides an average force on a particle so as to form a new equilibrium position between the half- and quarter-wave nodal positions. Since this is a stable equilibrium, no feedback control is required to achieve this positioning, and the 15 location of the average node is determined by the duty cycle of the switching waveform. This driving voltage can be written

$$
V(t)=A \sin \left(w_{1} t\right) S(t)+A \sin \left(w_{2} t\right)(1-S(t))
$$

where $A$ represents a voltage amplitude, and $S(t)$ is a unit amplitude switching function with variable mark to space ratio. It would also be possible to achieve this control by applying a waveform that is a sum of the two modal frequencies, and using amplitude weighting to effect control of the nodal position.

${ }^{20} V(t)=A_{1} \sin \left(w_{1} t\right)+A_{2} \sin \left(w_{2} t\right)$

The use of multiple frequencies within a single device has also been used in other circumstances ${ }^{28}$. To a first order approximation, the effect on the acoustic radiation force of combining two acoustic fields at different frequencies is linear - the resulting time averaged forces are the sum of those that would have acted if either frequency were present by itself. This is true both for when the frequency components are applied through a single transducer driven by a combined signal, or when two transducers are used (with the advantage 25 that each can be tuned to be resonant at its drive frequency). This can be shown by considering the expression for the time average second order pressure as used in derivations of radiation force by, for example, Yosioka and Kawasima ${ }^{45}$ or Gor'kov ${ }^{1}$. This is given by

$$
\left\langle p_{2}\right\rangle=\frac{1}{2 \rho_{0} c_{a}^{2}}\left\langle p_{1}^{2}\right\rangle-\frac{1}{2} \rho_{0}\left\langle v_{1}^{2}\right\rangle
$$

where $\rho_{0}$ is the quiescent fluid density, and $c_{a}$ is the speed of sound in the medium, $p_{l}$ and $v_{l}$ are the first order acoustic pressure and velocity, and the angled brackets denote time average. If $p_{l}$ and $v_{l}$ are each a sum of two sinusoids of different frequencies then the time 30 average of $p_{l}^{2}$ or $v_{l}^{2}$ is given by the sum of the time average of the independent components. A similar argument holds for the momentum flux terms that enter the radiation force derivations. It should be noted that this only applies when the time average is taken over many cycles - if the two frequencies are very close a zero-average beat frequency will be seen as described by Oberti et al. ${ }^{46}$, who also consider the case when two signals of the same frequency are superimposed.

Switching of frequencies has also been applied to the fractionation of particle suspensions. This was demonstrated by Mandralis et $35 \mathrm{al} .{ }^{47}$ in combination with a synchronised field-flow fractionation system, whereby particles were cycled away and towards a surface, with larger particles moving further and experiencing regions of higher flow.

Another fractionation method achieved by frequency switching and relying on response time of the particle has been proposed by Harris et al. ${ }^{48}$ which used a half-wavelength mode to position particles to a known location in the flow field, then by switching between the second and third modes particles are fractionated across the depth of the channel. Using continuous flow, different fractions can be 40 extracted using flow splitters. Similar approaches have been demonstrated experimentally in lateral devices ${ }^{49,50}$

\section{Applications}

An exhaustive description of the potential applications of planar ultrasonic resonators is beyond the scope of this article, but this section discusses a number of illustrative applications.

\section{Filtration, washing and separation}

45 Amongst the earliest applications for planar resonator ultrasonic particle manipulators was filtration. Some of these were on a relatively large, multiple wavelength scale ${ }^{51}$. The commercial Sonosep ${ }^{\mathrm{TM}}$ system, was described by Pui et al. ${ }^{52}$ as a component for bioreactor systems and production of therapeutic proteins. In their system, a planar resonator was used to aggregate hybridoma cells which then fall more rapidly under gravity, enhancing sedimentation and clarification stages.

However, working on a much smaller system, Hawkes et al. ${ }^{16}$ developed a filtration technique based on a half wave resonator of 
$250 \mu \mathrm{m}$ depth. They demonstrated the filter's ability to generate a particle depleted sample using yeast and latex particles of between 1.5 and $25 \mu \mathrm{m}$ diameter. A similar approach was taken by Harris et al. ${ }^{53}$, but in this case the channel was etched into Pyrex which was anodically bonded to a silicon substrate into which inlet and outlet channels had been anisotropically etched. Such microfabricated devices are expensive and complex to produce in small batches so there has been a recent tendency to use lower-cost disposable elements 5 such as capillaries ${ }^{54}$ which may then be integrated into a microfluidic system. Using disposable elements removes the need for expensive sterilisation processes between samples and prevents cross-contamination: essential to applications such as medical diagnosis and forensics. The flexibility of disposable or modular systems is also advantageous during the development of devices; microfluidic channels can be contained by large, inexpensive components making handling and modification more straightforward for the experimentalist.

10 As the physical properties of different populations of cells or particles vary, it is conceivable that they may be fractionated based on their acoustic properties or size, and therefore magnitude of the radiation force. An early illustration of this was reported by Johnson \& Feke ${ }^{55}$ by introducing a particle stream alongside a 'carrier fluid' stream to ensure the particles were constrained near the channel wall, so that on passing through a half-wavelength acoustic field particles then fractionated across channel width. The same group also demonstrated that it is possible to enhance separation of certain particle combinations by adjusting the properties of the carrying fluid ${ }^{56}$. 15 The use of a 'carrier fluid' or sheath flow within a planar resonator provides the potential to wash cells and particles, by moving them from the initial suspending fluid into the carrier fluid ${ }^{36}$. A limit on this, however, is the radiation force on the fluid/fluid boundary itself, as this can result in a mixing of the two fluid phases ${ }^{57}$.

\section{Sensing and detection}

The use of ultrasound to form a concentrated stream of particles can improve the sensitivity of a sensor. This is possible, either by 20 collecting the concentrated stream and removing excess fluid or by moving the plane of particles directly onto a sensor surface. The former approach, essentially a version of some of the filtration approaches described above, has been developed for biohazard detection by Townsend et al. ${ }^{39}$ who use a quarter-wavelength system to concentrate 1 micron sized spores close to the reflector surface with this concentrated region then extracted through one of two outlets, and separated from the remain clarified region. Quarter-wavelength systems, although typically less efficient than half-wavelength system, do allow the fluidic system to be simplified; as particles move to 25 the surface the flow only needs to be split into two streams to separate the concentrate from the clarified flow, as opposed to a halfwavelength where three are required to remove the clarified flow from both sides of the concentrate. The fluidic design is a continual challenge for planar flow-through systems; whilst the flow regime in such devices is highly laminar (and therefore predictable, and typically uniform) the acoustic field is less so and likewise the particle trajectories.

Hawkes et al. $^{58}$ developed a device to push bacterial spores directly onto antibody coated surface, and demonstrated a significant 30 improvement on the sensitivity and 200-fold increase in spore capture. In these sensing applications it is important for the interface to be less than a quarter wavelength from a pressure antinode in order to produce a net force towards the surface. The sensitivity, in particular, of the node location to the reflector thickness was demonstrated by Martin et al. ${ }^{38}$. This concept has also been combined with sensor elements, for example by Glynne-Jones et al. ${ }^{30}$ who designed a system incorporating an optical waveguide using a planar resonance to drive beads to the functionalised waveguide surface. On switching the excitation frequency, unbound beads can be driven away leaving 35 only the immobilised beads on the sensor surface, and therefore a means to improve the sensitivity of bead-based-bioassays.

Similar concepts have been explored to facilitate the measurement or monitoring of chemical reactions. For example, Radel et al. ${ }^{59}$ demonstrated the effective use of different excitation frequencies to control the movement of particles onto and away from an infrared spectroscopy probe for online periodic monitoring of chemical reactions, with potential application to bio-reactors. This multiplewavelength system, operating in the low $\mathrm{MHz}$ range, uses periodic frequency switching to control whether a positive or negative force is 40 seen at the probe surface, enabling particulates to be periodically screened by the probe, then returned to suspension and probe surface cleaned.

Chemical reactions can influence the acoustic properties of particulates, which can be an advantage for the purposes of sensing. This has been demonstrated by Kanazaki et al. ${ }^{60}$ monitoring ion-exchange processes. Replacement of counterions causes the water content of ion exchange resins to change, leading to a change in their acoustic properties and the resultant radiation force acting on a resin bead.

${ }_{45}$ Kanazaki et al. use a planar resonator to levitate a resin bead and the bead's location is determined by the balance between acoustic radiation forces and gravity. Following ion-exchange the bead is seen to relocate to a new equilibrium position with the displacement indicative of the fluid's ion content.

The relatively large scale of action of ultrasonic manipulation in comparison with other manipulation technologies means that there is an increasing interest in combining ultrasonic approaches with techniques such as dielectrophoresis ${ }^{61,62}$ or optical trapping ${ }^{63}$ within 50 integrated microfluidic systems.

\section{Cell-interaction studies and sonoporation}

Planar resonators are particularly suited to forming 2-d monolayers (and discoid aggregates) of cells, with much potential for future cellinteraction studies. Ultrasonic radiation forces provide a method that can produce useful forces on cells without adversely effecting viability $^{64-67}$, an aspect more thoroughly explored later in this series of tutorials. Additionally, due to the expansion of microsystem 55 technology and its synergy with ultrasonic resonators, the suitability of ultrasound devices for biological fluid processing and bio-sensing has attracted increasing interest.

Both Bazou et al. ${ }^{68}$ and Edwards et al. ${ }^{69}$ illustrate the formation of 2-d aggregates of hepatocyte cells in circular planar resonators. A 
uniform axial field causes the cells to move into a controlled plane free from the solid substrate, and such that significantly weaker lateral forces agglomerate the cells to form a closely packed monolayer of cells enabling cell-cell interaction and adhesion processes to be studied. This has also been demonstrated with neural cells ${ }^{70}$.

Where the lateral forces are strong enough, it is possible to introduce flow of a suitable medium as shown by Hultström et al. ${ }^{67}$. They 5 studied the effects of ultrasound exposure on adherent cell proliferation rate in a system that demonstrated the trapping of adherent cells in a 2-d agglomeration against fluid flow and isolated from the influence of any surfaces. Previously, Morgan et al. ${ }^{71}$ used a planar system combined with weaker lateral forces to increase mass transfer of toxicant to hepatocyte cells to expedite toxicological tests. In this work the lateral force also improves formation of spheroidal clusters where cells move out of plane creating 3-d constructs, conducive to mimicking in-vivo hepatocyte cell function. Similarly, the introduction of fluid flow also serves to mimic the action of 10 blood where Bazou et al. ${ }^{72}$ used this to investigate the development of cancer cells clusters, again for toxicological assessment. An extension of this concept is to immobilise these cell constructs in a gel matrix as shown by Gherardini et al. ${ }^{8}$ using yeast cells, but more recently by Bazou et al. ${ }^{73,74}$ using discoids of an hepatocyte cell line immobilised in alginate for rapid creation of in-vitro tissue models.

There has been much research on the use of ultrasound to transfect cells (sonoporation) to permit bio-molecules to cross the cell membrane for example to effect toxicological analysis or gene therapy. Some of these studies use a standing wave field, specifically 15 with planar resonators. Although the mechanisms are unclear, there is evidence to suggest that these systems have the potential to produce high transfection rates and improve viability over that reported using inertial cavitation ${ }^{75-77}$. Whilst the potential for poration in planar resonators is promising ${ }^{78}$ there still remains little evidence of the cell physiology, cell dynamics, acoustic environment and poration mechanism in such systems.

\section{Conclusions}

${ }_{20}$ Particle and cell manipulation using ultrasonic standing waves is well matched to lab-on-a-chip technology and of the various techniques available to the researcher for generating an appropriate field, the planar layered resonator remains the most straightforward. Its simplicity lends itself to modeling and optimization using highly efficient one dimensional models, but the results of these need to be considered along with two and three dimensional representations, due to the importance of lateral field variations in many devices.

\section{References}

25 1. L. P. Gor'kov, Sov. Phys. Dokl., 1962, 6, 773-775.

2. T. Lilliehorn, U. Simu, M. Nilsson, M. Almqvist, T. Stepinski, T. Laurell, J. Nilsson and S. Johansson, Ultrasonics, 2005, 43, 293-303.

3. H. M. Hertz, Journal Of Applied Physics, 1995, 78, 4845-4849.

4. J. Lee, S.-Y. Teh, A. Lee, H. H. Kim, C. Lee and K. K. Shung, Ultrasound in medicine \& biology, 2010, 36, 350-355.

5. P. Augustsson, J. Persson, S. Ekstrom, M. Ohlin and T. Laurell, Lab on a Chip, 2009, 9, 810-818.

30 6. F. Petersson, A. Nilsson, C. Holm, H. Jonsson and T. Laurell, Lab On A Chip, 2005, 5, 20-22.

7. G. Goddard and G. Kaduchak, Journal of the Acoustical Society of America, 2005, 117, 3440-3447.

8. L. Gherardini, C. M. Cousins, J. J. Hawkes, J. Spengler, S. Radel, H. Lawler, B. Devcic-Kuhar and M. Groschl, Ultrasound In Medicine And Biology, 2005, 31, 261-272.

9. A. Haake, A. Neild, G. Radziwill and J. Dual, Biotechnology And Bioengineering, 2005, 92, 8-14.

35 10. A. Neild, S. Oberti, F. Beyeler, J. Dual and B. J. Nelson, Journal Of Micromechanics And Microengineering, 2006, 16, 1562-1570.

11. M. Bok, H. Y. Li, L. Y. Yeo and J. R. Friend, Biotechnology And Bioengineering, 2009, 103, 387-401.

12. J. Shi, X. Mao, D. Ahmed, A. Colletti and T. J. Huang, Lab on a Chip, 2008, 8, 221 - 223.

13. C. D. Wood, J. E. Cunningham, R. O'Rorke, C. Walti, E. H. Linfield, A. G. Davies and S. D. Evans, Applied Physics Letters, $2009,94$.

14. V. Yantchev, J. Enlund, I. Katardjiev and L. Johansson, Journal Of Micromechanics And Microengineering, 2010, $20,035031$.

40 15. M. Gröschl, Acustica, 1998, 84, 432-447.

16. J. J. Hawkes and W. T. Coakley, Sensors and Actuators B-Chemical, 2001, 75, 213-222.

17. M. Hill, Y. Shen and J. J. Hawkes, Ultrasonics, 2002, 40, 385-392.

18. Y. Abe, M. Kawaji and T. Watanabe, Exp. Therm. Fluid Sci., 2002, 26, 817-826.

19. C. Courtney, C.-K. Ong, B. Drinkwater, P. Wilcox, C. Démoré, S. Cochran, P. Glynne-Jones and M. Hill, Journal of the Acoustical Society of 45 America, 2010, 128, EL195-199

20. H. Nowotny, E. Benes and M. Schmid, Journal of the Acoustical Society of America, 1991, 90, 1238-1245.

21. P. D. Wilcox, R. S. C. Monkhouse, P. Cawley, M. J. S. Lowe and B. A. Auld, NDT E Int., 1998, 31, 51-64.

22. M. Gröschl, Acustica, 1998, 84, 632-642.

23. H. Bruus, Lab on a Chip, 2012, 12, 20-28.

50 24. S. Sherrit, Leary, S., Dolgin, B., Bar-Cohen, Y., Comparison of the Mason and KLM Equivalent Circuits for Piezoelectric Resonators in the Thickness Mode, Nevada USA, 1999.

25. J. Dual and D. Möller, Lab on a Chip, 2012.

26. Krimholt.R, D. A. Leedom and G. L. Matthaei, Electron Lett, 1970, 6, 398-\&.

27. R. J. Townsend, M. Hill, N. R. Harris and N. M. White, Ultrasonics, 2006, 44, e467-e471.

55 28. O. Manneberg, J. Svennebring, H. M. Hertz and M. Wiklund, Journal Of Micromechanics And Microengineering, $2008,18$.

This journal is (C) The Royal Society of Chemistry [year] $\quad$ Journal Name, [year], [vol], 00-00 | 7


29. S. M. Hagsater, T. G. Jensen, H. Bruus and J. P. Kutter, Lab on a Chip, 2007, 7, 1336-1344.

30. P. Glynne-Jones, R. J. Boltryk, M. Hill, F. Zhang, L. Q. Dong, J. S. Wilkinson, T. Melvin, N. R. Harris and T. Brown, Anal. Sci., $2009,25,285-291$.

31. S. M. Hagsater, A. Lenshof, P. Skafte-Pedersen, J. P. Kutter, T. Laurell and H. Bruus, Lab on a Chip, 2008, 8, 1178-1184.

32. O. Manneberg, B. Vanherberghen, B. Onfelt and M. Wiklund, Lab on a Chip, 2009, 9, 833-837.

5 33. I. Gonzalez, L. J. Fernandez, T. E. Gomez, J. Berganzo, J. L. Soto and A. Carrato, Sensors And Actuators B-Chemical, $2010,144,310-317$.

34. J. J. Hawkes, M. Gröschl, E. Benes, H. Nowotny and W. T. Coakley, Revista de Acustica, 2002, 33, PHA-01-007-IP.

35. J. J. Hawkes, D. Barrow and W. T. Coakley, Ultrasonics, 1998, 36, 925-931.

36. J. J. Hawkes, R. W. Barber, D. R. Emerson and W. T. Coakley, Lab On A Chip, 2004, 4, 446-452.

37. M. Hill, R. J. Townsend and N. R. Harris, Ultrasonics, 2008, 48, 521-528.

10 38. S. P. Martin, R. J. Townsend, L. A. Kuznetsova, K. A. J. Borthwick, M. Hill, M. B. McDonnell and W. T. Coakley, Bio-sensors and Bioelectronics, 2005, 21, 758-767.

39. R. J. Townsend, M. Hill, N. R. Harris and M. B. McDonnell, Ultrasonics, 2008, 48, 515-520.

40. P. Glynne-Jones, R. J. Boltryk, M. Hill, N. R. Harris and P. Baclet, J. Acoust. Soc. Am., 2009, 126, EL75-EL79.

41. T. Kozuka, T. Tuziuti, H. Mitome and T. Fukuda, Jpn. J. Appl. Phys. Part 1 - Regul. Pap. Short Notes Rev. Pap., 1998, 37, $2974-2978$.

15 42. C. Demore, Q. Yongqiang, S. Cochran, P. Glynne-Jones, Y. Congwei and M. Hill, 2010 International Ultrasonics Symposium, 2010.

43. T. L. Tolt and D. L. Feke, Chemical Engineering Science, 1992, 48, 527-540.

44. P. Glynne-Jones, R. J. Boltryk, N. R. Harris, A. W. J. Cranny and M. Hill, Ultrasonics, 2010, 50, 68-75.

45. K. Yosioka and Y. Kawasima, Acustica, 1955, 5, 167-173.

46. S. Oberti, A. Neild and J. Dual, Journal of the Acoustical Society of America, 2007, 121, 778-785.

20 47. Z. Mandralis, W. Bolek, W. Burger, E. Benes and D. L. Feke, Ultrasonics, 1994, 32, 113-121.

48. N. Harris, R. Boltryk, P. Glynne-Jones and M. Hill, Physics Procedia, 2010, 3, 277-281.

49. K. M. Lim and Y. Liu, Lab on a Chip, 2011, 11, 3167-3173.

50. F. Petersson, PhD PhD Thesis, Lund University, 2007.

51. S. Gupta and D. L. Feke, Aiche Journal, 1998, 44, 1005-1014.

25 52. P. W. S. Pui, F. Trampler, S. A. Sonderhoff, M. Groeschl, D. G. Kilburn and J. M. Piret, Biotechnology Progress, 1995, 11, $146-152$.

53. N. R. Harris, M. Hill, S. P. Beeby, Y. Shen, N. M. White, J. J. Hawkes and W. T. Coakley, Sens. Actuator B-Chem, $2003,95,425-434$.

54. B. Hammarstrom, M. Evander, H. Barbeau, M. Bruzelius, J. Larsson, T. Laurell and J. Nillsson, Lab on a Chip, 2010, 10, $2251-2257$.

55. D. A. Johnson and D. L. Feke, Separations Technology, 1995, 5, 251-258.

56. S. Gupta, D. L. Feke and I. Manaszloczower, Chemical Engineering Science, 1995, 50, 3275-3284.

30 57. L. Johansson, S. Johansson, F. Nikolajeff and S. Thorslund, Lab on a Chip, 2009, 9, 297-304.

58. J. J. Hawkes, M. J. Long, W. T. Coakley and M. B. McDonnell, Biosensors and Bioelectronics, 2004, 19, 1021-1028.

59. S. Radel, M. Brandstetter and B. Lendl, Ultrasonics, 2010, 50, 240-246.

60. T. Kanazaki, S. Hirawa, M. Harada and T. Okada, Analytical Chemistry, 2010, 82, 4472-4478.

61. S. K. Ravula, D. W. Branch, C. D. James, R. J. Townsend, M. Hill, G. Kaduchak, M. Ward and I. Brener, Sensors and Actuators B-Chemical, 2008, 130, 645-652.

62. M. Wiklund, C. Günther, R. Lemor, M. Jäger, G. Fuhr and H. M. Hertz, Lab on a Chip, 2006, 6, 1537-1544.

63. G. Thalhammer, R. Steiger, M. Meinschad, M. Hill, S. Bernet and M. Ritsch-Marte, Biomed. Opt. Express, 2011, 2, $2859-2870$.

64. H. Bohm, P. Anthony, M. R. Davey, L. G. Briarty, J. B. Power, K. C. Lowe, E. Benes and M. Groschl, Ultrasonics, $2000,38,629-632$.

65. D. Bazou, R. Kearney, F. Mansergh, C. Bourdon, J. Farrar and M. Wride, Ultrasound Med Biol, 2011, 37, 321-330.

40 66. B. Vanherberghen, O. Manneberg, A. Christakou, T. Frisk, M. Ohlin, H. M. Hertz, B. Onfelt and M. Wiklund, Lab on a Chip, $2010,10,2727-2732$.

67. J. Hultström, O. Manneberg, K. Dopf, H. M. Hertz, H. Brismar and M. Wiklund, Ultrasound Med. Biol., 2006, 33, 175-181.

68. D. Bazou, G. P. Dowthwaite, I. M. Khan, C. W. Archer, J. R. Ralphs and W. T. Coakley, Mol Membr Biol, 2006, 23, 195-205.

69. G. O. Edwards, D. Bazou, L. A. Kuznetsova and W. T. Coakley, Cell Commun Adhes, 2007, 14, 9-20.

70. D. Bazou, G. A. Foster, J. R. Ralphs and W. T. Coakley, Molecular Membrane Biology, 2005, 22, 229-240.

45 71. J. Morgan, J. F. Spengler, L. Kuznetsova, W. T. Coakley, J. Xu and W. M. Purcell, Toxicol. Vitro, 2004, 18, 115-120.

72. D. Bazou, M. J. Santos-Martinez, C. Medina and M. W. Radomski, Br J Pharmacol, 2011, 162, 1577-1589.

73. D. Bazou, W. T. Coakley, A. J. Hayes and S. K. Jackson, Toxicol In Vitro, 2008, 22, 1321-1331.

74. D. Bazou, Cell Biol Toxicol, 2010, 26, 127-141.

75. Y. H. Lee and C. A. Peng, Gene Ther, 2005, 12, 625-633.

50 76. Y. H. Lee and C. A. Peng, Ultrasound Med Biol, 2007, 33, 734-742.

77. S. Rodamporn, N. R. Harris, S. P. Beeby, R. J. Boltryk and T. Sanchez-Elsner, IEEE Trans Biomed Eng, 2011, 58, $927-934$.

78. D. Carugo, D. N. Ankrett, P. Glynne-Jones, L. Capretto, R. J. Boltryk, X. Zhang, P. A. Townsend and M. Hill, Biomicrofluidics, 2011, In Press. 


\section{Figures}

Fig. 1 Layered components of a planar resonator

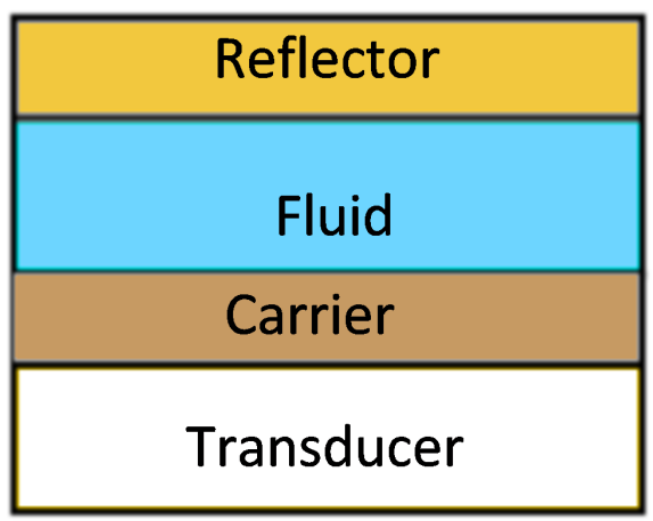


Fig. 2 Mason model configuration (after Sherrit et al. ${ }^{24}$ ). The top-left and -right ports represent the acoustic connections to subsequent 5 layers (acoustic pressure represented as a voltage), and the bottom port the electrical connections to the transducer.

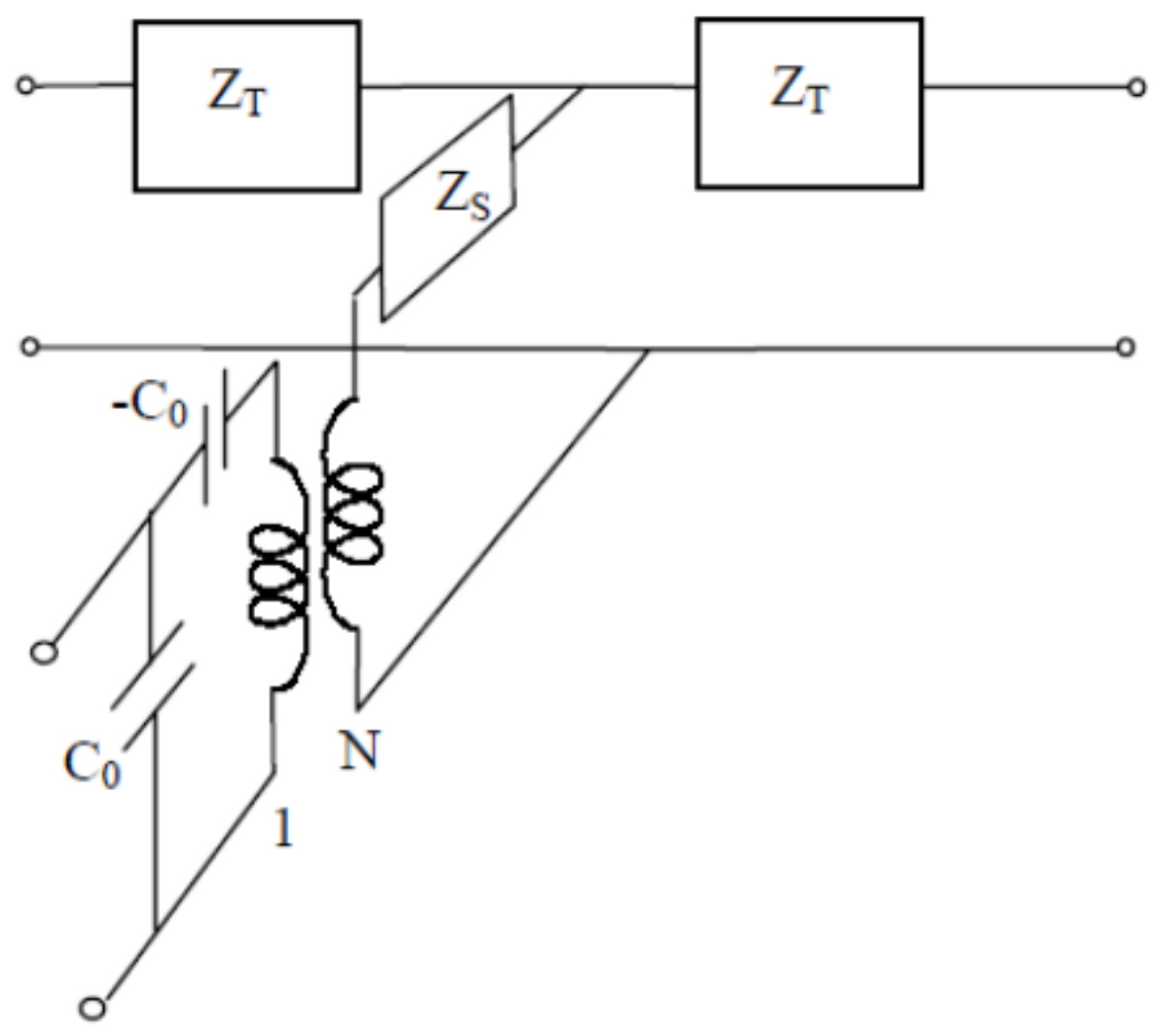


Fig. 3 FEA model compared to transfer impedance model of a layered resonator for a typical quarter wave configuration. The graph shows how the energy density in the fluid layer varies with excitation frequency.

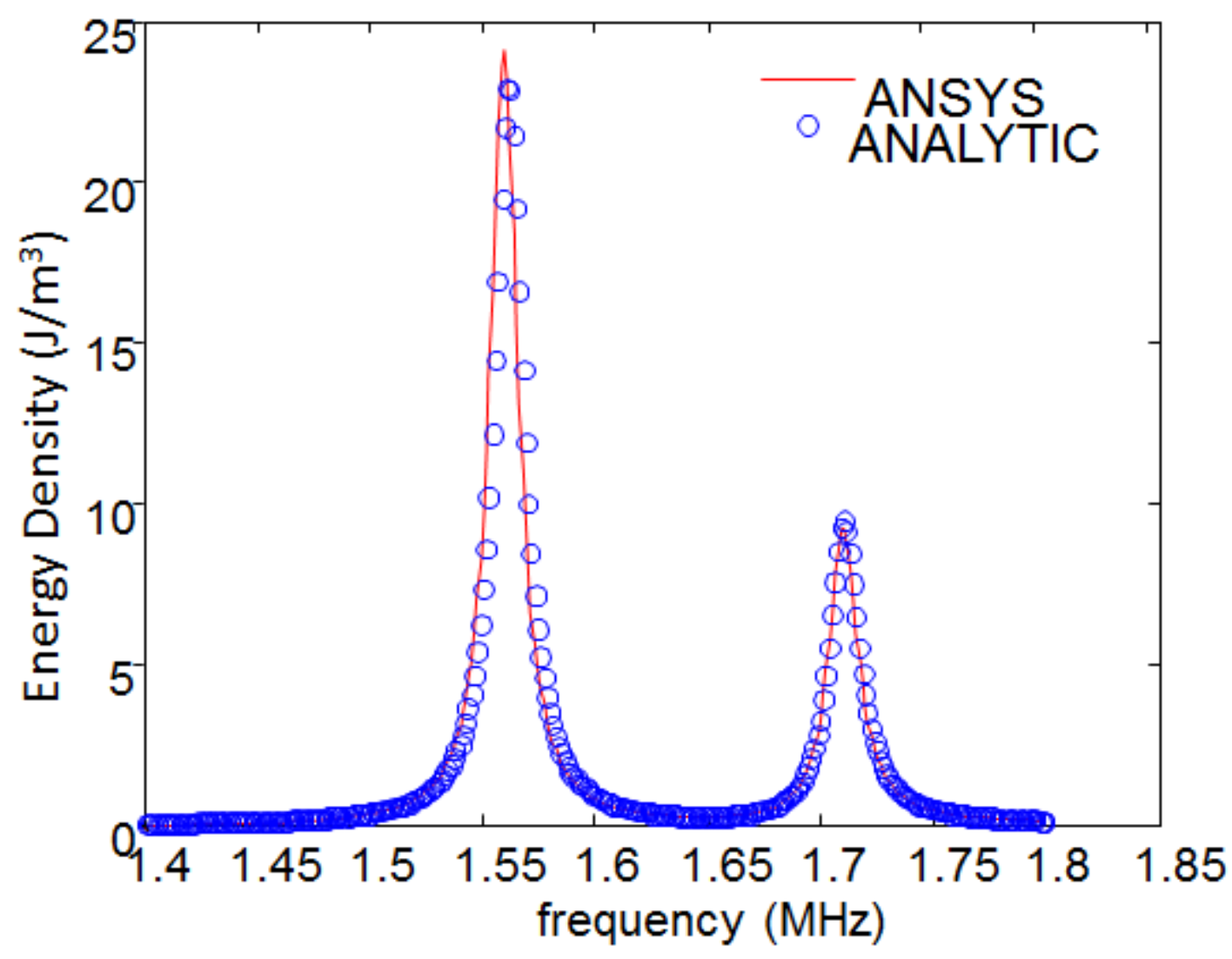

15 
Fig. 4 (a) Modelled acoustic pressure amplitude distribution within a particle separator shows non-ideal behaviour (arbitrary scale: black - maximum; white,zero) (Townsend et al. ${ }^{27}$ ). This is a modal analysis with the carrier layer replaced by a rigid boundary condition. The white pressure node can be seen to be corrugated in shape; the acoustic radiation forces (proportional to the spatial gradient of the 5 pressure amplitude squared) can be seen to be variable across the device width and weaker near the right hand edge. (b) Cross sectional image taken from the quarter-wave device described by Glynne-Jones et al. ${ }^{44}$ showing the effect of a similar field on a population of $10 \mu \mathrm{m}$ polystyrene beads as they flow through the device; Corrugation and a weaker field near the edge can be seen.

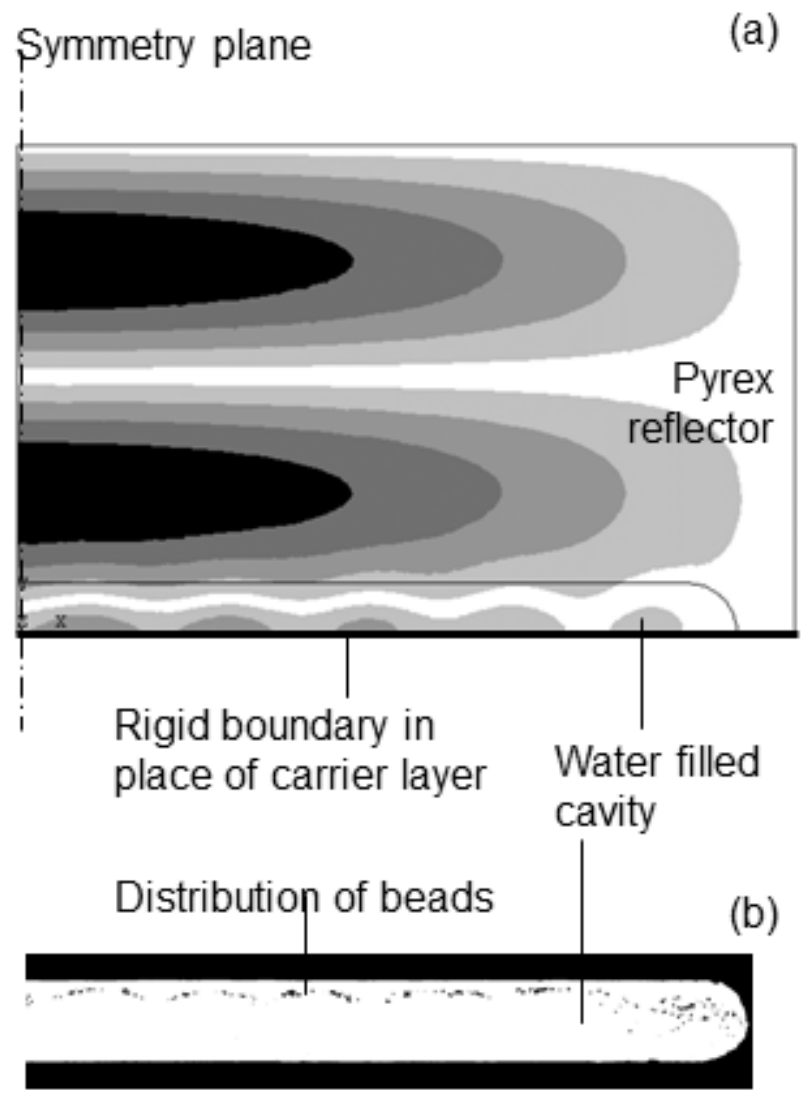


Fig. 5 Typical pressure amplitude distributions in (a) half-wave, (b) inverted quarter-wave, (c) quarter-wave, (d) thin-reflector resonators. The differing positions of the pressure nodes yield various behaviours.

10

(a)

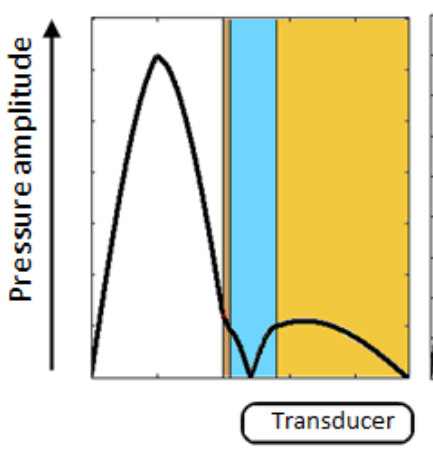

(b)

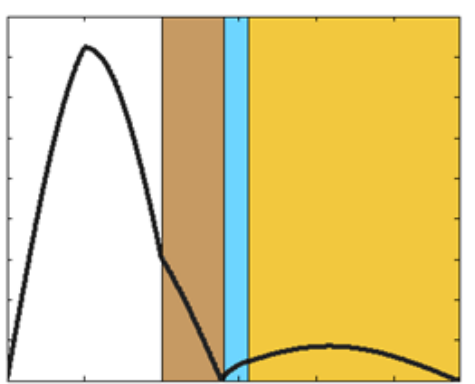

Carrier (c)

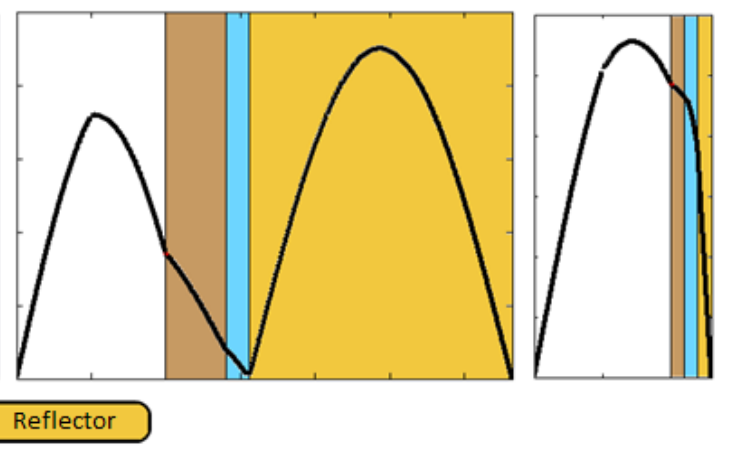

15 
Fig. 6 Parameter space optimisation for a half-wave resonator. Maximum forces on a 10um polystyrene bead for (a) combinations of fluid and carrier layer thickness with a $\lambda / 4$ reflector thickness, (b) combinations of fluid and reflector layer thicknesses, (c) Force on a $10 \mu \mathrm{m}$ polystyrene bead at reflector/fluid boundary, (d) Performance number for combinations of fluid and reflector layer thicknesses. 5 (b)-(d) are for zero carrier-layer thickness; the transducer thickness is $1 \mathrm{~mm}$.
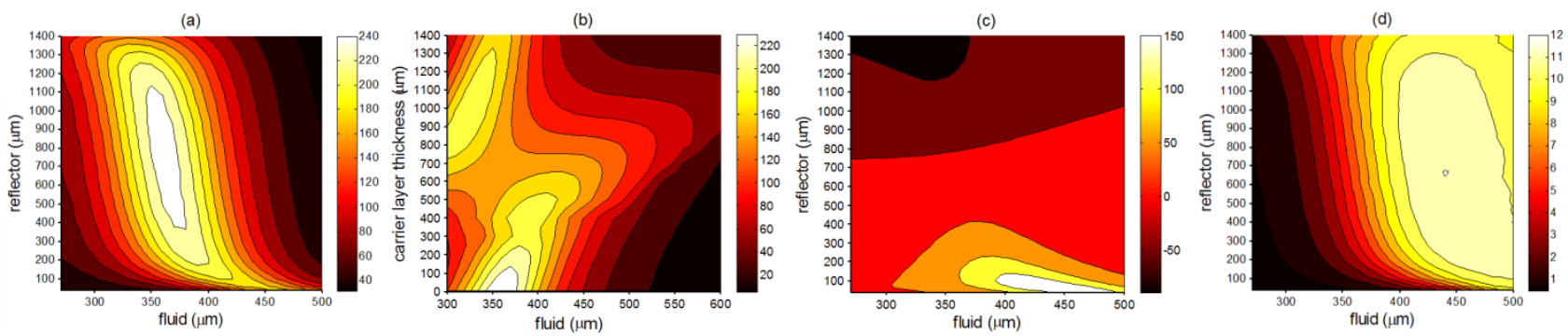
Fig. 7 Examining the resonances found in the configuration with the optimum combination of layer thicknesses. (a) Two energetic frequencies can be seen corresponding to half-wave resonances with differing force distributions across the fluid layer. (b),(c) The pressure amplitude distributions at these two frequencies. Dotted lines indicate the boundaries between the transducer, fluid and reflector 5 layers (transducer on the left).

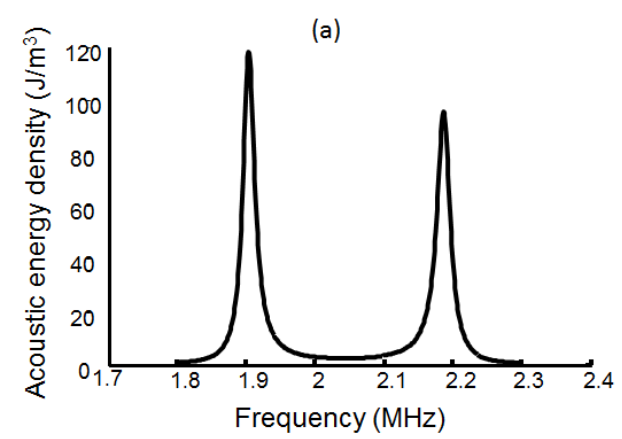

(b)
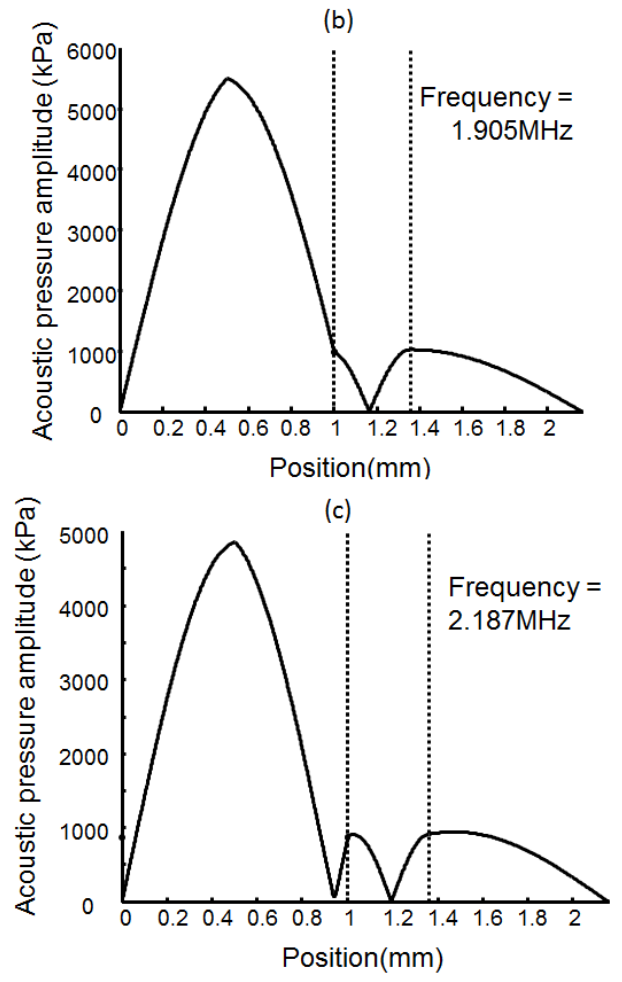
Fig. 8 Parameter space optimisation for a thin-reflector resonator. (a) Average radiation force in the fluid layer on a $10 \mu \mathrm{m}$ polystyrene bead. (b) Performance number. In both cases the transducer thickness is $1 \mathrm{~mm}$, and there is no carrier layer.
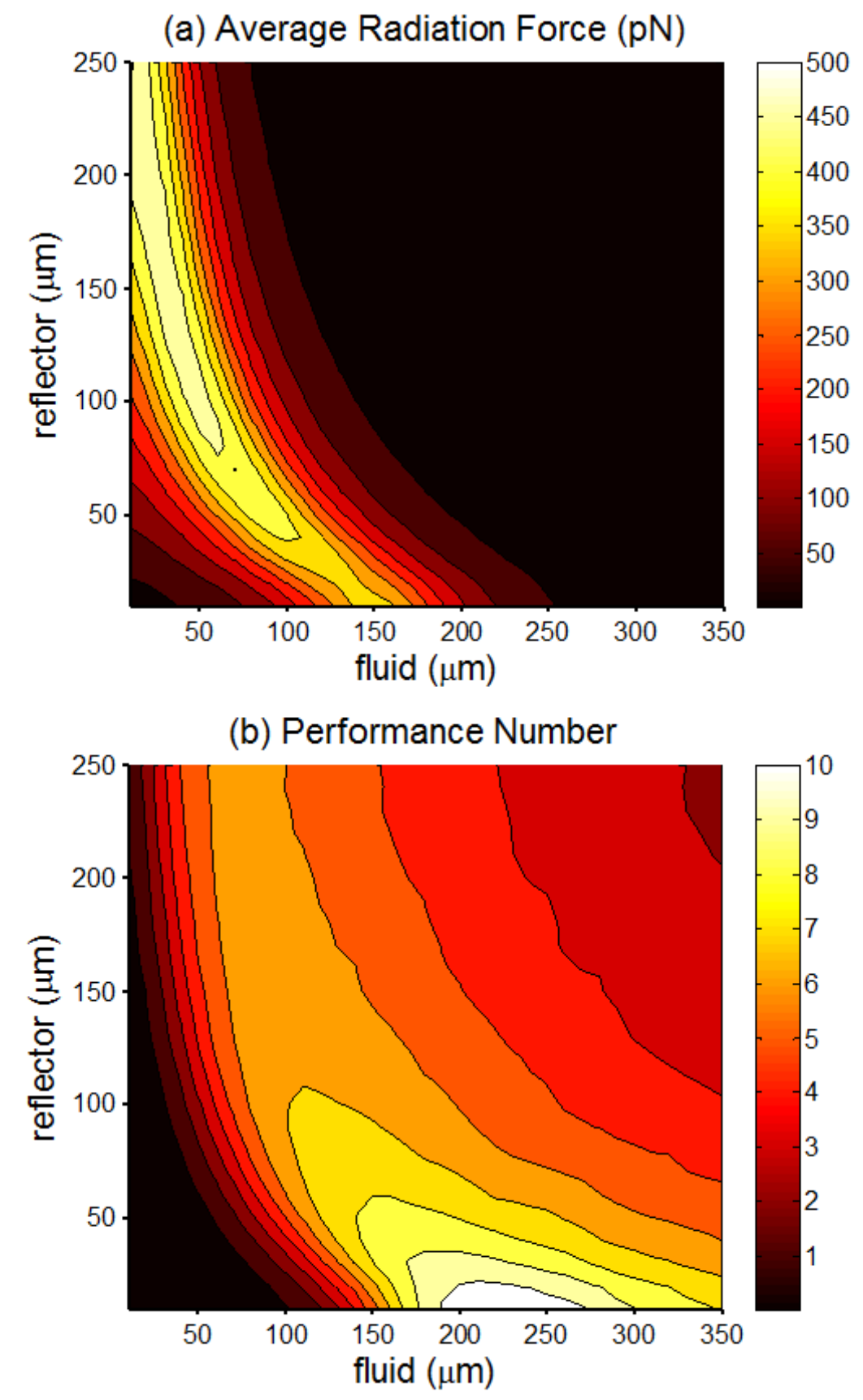


\section{Author Biographies:}

Peter Glynne-Jones graduated with first class honours from the School of Electronics and Computer Science at the University of Southampton. He was sponsored through his degree by the Defence Education and Science Group and was an IEE scholar. He was awarded a Ph.D., "Vibration powered generators for self-powered microsystems", from ${ }_{5}$ Southampton in 2001.

More recently, Peter has been involved in researching the manipulation of particles in microsystems using ultrasonic radiation forces, covering topics from fundamental modelling of systems, through manipulation, separation and sorting devices. His current focus extends to the applications of ultrasonic radiation forces in biological assays, and tissue engineering.

Dr. Rosemary Boltryk is a lecturer within Engineering Sciences at the University of Southampton. She completed a PhD in acoustic particle manipulation and maintains an interest in its bio-medical application and aspects of microfluidic design. She was involved the microfluidic development of a DNA bio-sensing to identify susceptibility to breast cancer. More recent work has involved the acoustic and fluidic design of acoustic particle manipulators for bio-sensing 15 applications. She has received EPSRC (UK) funding to investigate the use of micro-streaming flows to facilitate biomedical studies on cardiac cells and is a Co-Investigator on the Sonotweezers project simulating the effect of acoustics in cell forces and streaming in the surrounding fluid. She is Chartered Engineer and a member of the IMechE.

Martyn Hill is Professor of Electromechanical Systems and Head of Engineering Sciences at the University of Southampton. Martyn was awarded a BSc from the Institute of Sound and Vibration Research at Southampton and then 20 moved to the mechanical engineering department where he carried out research into parameter estimation techniques for noninvasive biomedical measurement and the development of automotive sensors. He is now a Professor in the ElectroMechanical Research Group with principle research interests in the use and modelling of ultrasonic radiation forces, particularly their application in microfluidic systems, and in the manipulation of micron scale particles and cells. 
